\title{
Overview and performance of the D-Egg optical sensor for IceCube-Gen2
}

\section{The IceCube-Gen2 Collaboration ${ }^{\dagger}$, S. Shimizu ${ }^{1}$}

${ }^{\dagger}$ http://icecube.wisc.edu/collaboration/authors/icrc17_gen2

${ }^{1}$ Nippon Marine Enterprise, Yokosuka, Kanagawa 238-0004, Japan

E-mail: ayadhepburn.s.chiba-u.ac.jp

IceCube-Gen2, a future upgrade of the IceCube Neutrino Observatory, is designed to enhance our ability to detect neutrinos above $10 \mathrm{TeV}$ via a significant increase in instrumented volume compared to IceCube. We expect an increased astrophysical neutrino detection rate of up to an order of magnitude with IceCube-Gen2, using a combination of more instrumentation, larger string spacing, and enhanced optical sensor performance. A new optical sensor module, the Dual optical sensor in an Ellipsoid Glass for Gen2 (called "D-Egg"), which houses two 8" HQE photomultiplier tubes (PMTs) in a UV-transparent pressure-resistant glass with an optical coupling elastomer, significantly improves photon detection efficiency in all directions. Its elongated mechanical shape is optimized for cost-effective instrumentation of optical modules and high photon transmittance to PMTs. Furthermore, it is optimized to endure high pressures, as the ice refreezing period during detector deployment requires the module to withstand pressures up to 70 MPa. Additionally, D-Egg's readout system takes advantage of advances in embedded computing power, enabling simpler, more efficient event triggering and seamless event recording compared to IceCube. Herein, we report on the design and properties of D-Egg's components. A series of laboratory measurements and detailed comparisons with the simulated design indicate that D-Egg provides a technological solution for IceCube-Gen2.

Corresponding Author: A. Ishihara ${ }^{*, 2}$, A. Stoessl ${ }^{2}$ and S. Yoshida ${ }^{2}$

${ }^{2}$ Dept. of Physics and Institute for Global Prominent Research, Chiba University, Chiba $263-$ 8522, Japan

35th International Cosmic Ray Conference -ICRC2017-

10-20 July, 2017

Bexco, Busan, Korea

*Speaker. 


\section{Overview of D-Egg}

The IceCube Digital Optical Module (DOM) is a successful, highly reliable optical sensor unit, which has been running for more than 10 years with an extremely small $(\leq 0.5 \%)$ post-deployment failure rate. The IceCube deep ice array consists of 5160 DOMs and has enabled the observation of rare high-energy neutrino events that consist of TeV-PeV cosmic neutrino fluxes over the the background of atmospheric muons and neutrinos. The next generation experiment, IceCube-Gen2, enhances the system's effective detection volume, with string separation distances increased by a factor of two and a number of strings comparable to the current IceCube detector; Gen2 also uses the ice $90 \mathrm{~m}$ above and $170 \mathrm{~m}$ below the IceCube detector. This design presents the significant challenge of achieving improved performance with fewer strings in a given area. The D-Egg optical sensor module was developed to overcome this difficult challenge.

An overview of the D-Egg's design is presented in Fig. 1. Two 8" Hamamatsu R5912-100 HQE photomultiplier tubes (PMTs) are enclosed in an elongated UV-transparent pressure-resistant housing that is $305 \mathrm{~mm}$ in diameter, in contrast with the current DOM, which consists of a single downward-facing 10" Hamamatsu R7081-02 PMT in a spherical housing. The 10\% reduction in diameter compared to the IceCube DOM reduces the fuel needed for drilling to a depth of $2620 \mathrm{~m}$ by $20 \%$. The shape and material of the D-Egg's pressure vessel have been carefully designed to optimize Cherenkov photon detection as well as mechanical strength, which is required for stabilization against the maximum pressure of $70 \mathrm{MPa}$ during the hole refreezing period. Minimization of noise expected from the glass material is also investigated. The detection of short-wavelength (UV) photons is important for Cherenkov detectors because the Cherekov radiation has $1 / \lambda$ dependence. The PMTs are optically coupled to the housing glass with a silicone elastomer that also holds the upward- and downward-facing PMTs. The PMT base, called the high-voltage (HV) board, consists of the HV module and PMT signal pulse-shaping circuits and is attached to each
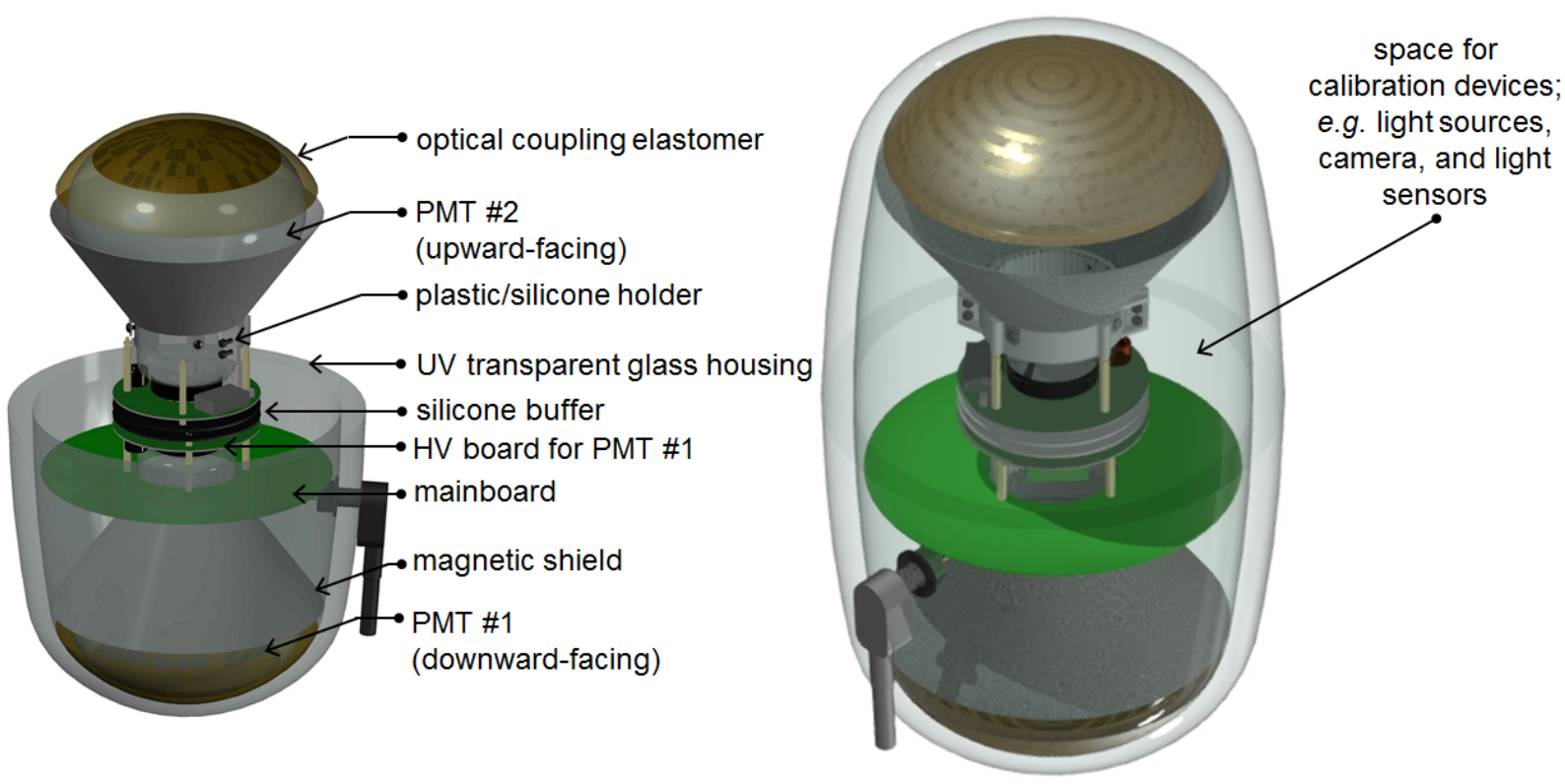

Figure 1: Structural overview of the D-Egg. 
PMT. The readout, control, and communication circuits are on the mainboard. The mainboard is placed at the neck of the downward-facing PMT with an internal structure covering the PMT neck, though the mainboard position is adjustable. A silicone buffer structure to connect the lower and upper PMTs is sandwiched between the HV boards. An open space near the upward-facing PMT can be used to install additional calibration devices such as the calibrated light sources [1] or the ice camera [2].

The D-Egg has been developed for increasing effective photocathode area with an upwardfacing PMT that also gives an additional directional sensitivity [3], while keeping as much as possible of the successful IceCube DOM design. Other innovative optical sensors are also under investigation for the Gen2 detector, such as the PDOM [4], the MDOM [5] and the WOM [6].

\section{Housing}

We have determined the optimal glass thickness for photon detection and mechanical strength to be $10 \mathrm{~mm}$ for the top and bottom of the glass sphere, where the PMT is sensitive, and $20 \mathrm{~mm}$ for the side of the housing, to enhance mechanical strength. A series of pressure tests up to 70 MPa have been performed on the prototype D-Egg glass housing with and without the enclosed PMTs in the high-pressure tank at JAMSTEC ${ }^{1}$ in Yokosuka, Japan. We measured the mechanical displacement of the glass under high pressure using three strain gauges (Kyowa KFG Series ${ }^{2}$ ) attached to the inside of the housing glass. The experimental results were compared with those from a simulation, as presented in the bottom panel of Fig. 2. The calculated strain $\varepsilon$, displacement per unit length, and measured strain show reasonable agreement, except that the measured strain was smaller than the simulated strain by a factor of two near the top of the housing. While this is conservative, we expect the difference to be due to a slight misalignment of the strain gauge, rather than to an inaccuracy in the calculation.

Optical transparency is a key feature of the D-Egg glass. The new glass material was developed by Okamoto Glass ${ }^{3}$ for high UV light transmittance and low radioactive noise. There is a strong correlation between light transmittance for wavelengths shorter than $400 \mathrm{~nm}$ and the $\mathrm{Fe}$ content of the glass. Table 1 shows this correlation and indicates that an $\mathrm{Fe}_{2} \mathrm{O}_{3}$ content of no more than $0.006 \%$ by weight is required to achieve the target UV light detection efficiency ( $\geq 70 \%$ at $320 \mathrm{~nm}$ ). While the glass batch (the raw materials mixture for glass melting) itself was optimized for low $\mathrm{Fe}$ content, when we measured the $\mathrm{Fe}_{2} \mathrm{O}_{3}$ content after glass construction with an X-ray fluorescence spectrometer (XRF), we found that the $\mathrm{Fe}_{2} \mathrm{O}_{3}$ content differed for glass samples with different construction methods. We observed an enhancement of Fe content from the batch containing samples indicated as H-3-G1 and H-3-G4 in Table 1. Further study identified that the process involving the use of "cullet", which is broken glass from previous glass production, led to Fe contamination from the older glass and the processes involved in cleaning and storing the cullet. An updated production scheme achieved transmittance of $75 \%$ at $10 \mathrm{~mm}$ after reflection correction, which is a significant improvement over the IceCube glass, as seen in Fig. 3.

\footnotetext{
${ }^{1}$ Japan Agency for Marine-Earth Science and Technology; http://www.jamstec.go.jp/e

${ }^{2} \mathrm{http}$ ///www. kyowa-ei.com/eng/product/category/strain_gages/kfg

${ }^{3}$ http://www. okamoto-glass. co.jp/eng/
} 

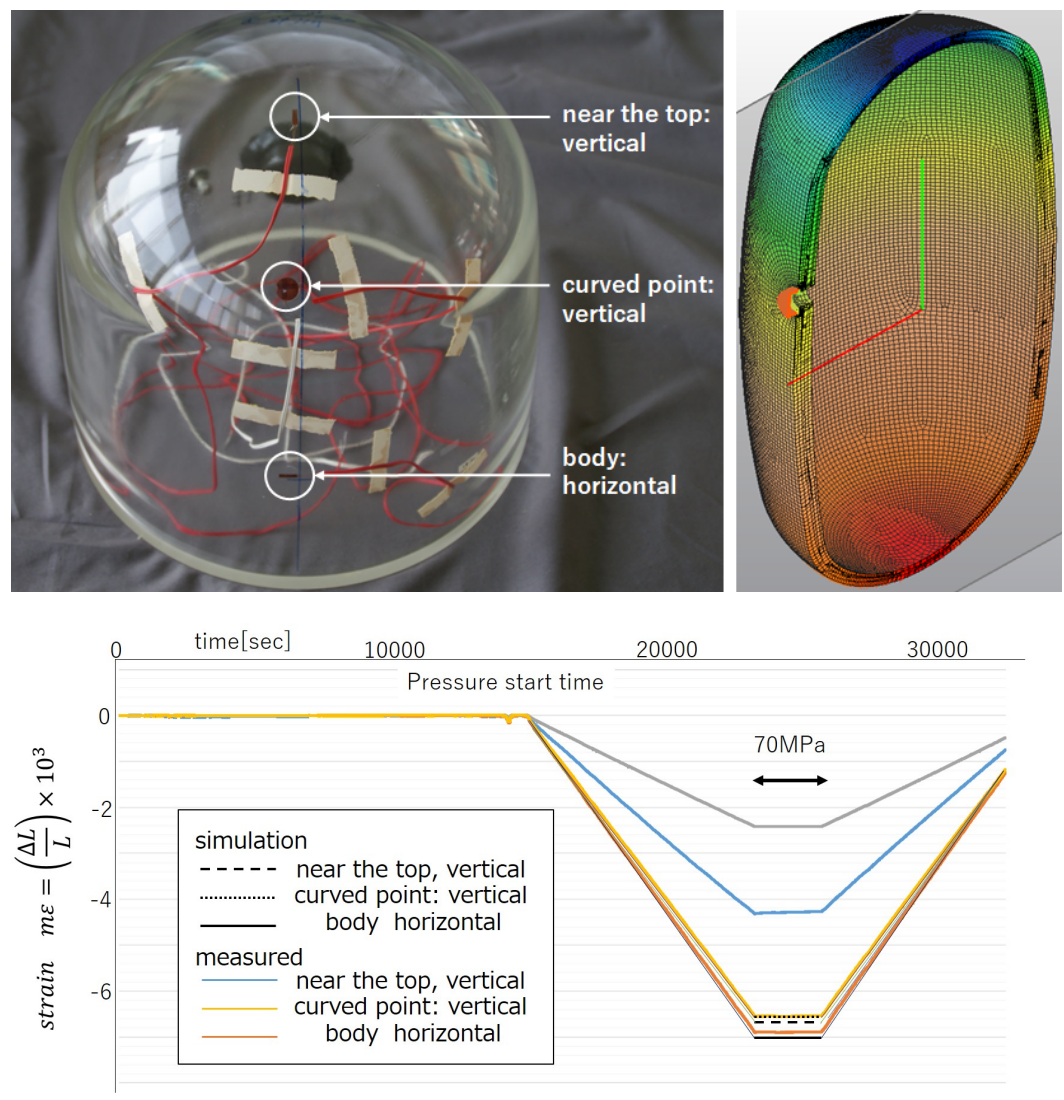

Figure 2: The setup (top, left), vertical displacement simulation (top, right) and strain gauge measurement results (bottom) of glass under $70 \mathrm{MPa}$ pressure.

The PMTs are held and optically coupled by a silicone elastomer to the glass housing. The thickness of the optical coupling elastomer is $5 \mathrm{~mm}$, which is a factor of two reduction compared to that of the IceCube DOM, enabled by matching the glass curvature with the cathode surface curvature of the PMT. In addition to the light transmittance of the elastomer, its hardness is also important, since it must provide good mechanical support for the PMTs while also being soft enough to firmly adhere to the glass. Therefore, two silicone materials were developed for the D-Egg by Shinetsu Silicone ${ }^{4}$ for the best UV transmittance and optimized hardness, as shown in the right panel of Fig. 3 and in Table 2. Both materials exceed a transmittance of $90 \%$ at $300 \mathrm{~nm}$, indicating that only a small effect is expected from UV absorption in the elastomer. The IceCube gel referenced in the figure is RTV6136-D1 (General Electric), and later, a similar formulation from Quantum Silicones that has a much softer texture.

The majority of dark noise in the IceCube DOM originates from its glass housing [7]. Cherenkov light from the radioactive decay of $\mathrm{K}_{40}$ significantly contributes to the Poissonian noise component as well as to noise from scintillation in the glass. The IceCube glass sphere contains a $\mathrm{K}_{2} \mathrm{O}$ concentration of $\approx 0.03 \%$ by weight, which roughly corresponds to $100 \mathrm{~Bq}$ of beta decays per sphere. With a similar $\mathrm{K}_{2} \mathrm{O}$ concentration, the effective noise per PMT can be reduced, because the amount

\footnotetext{
${ }^{4} \mathrm{http} / / / \mathrm{www}$. shinetsusilicones. com
} 

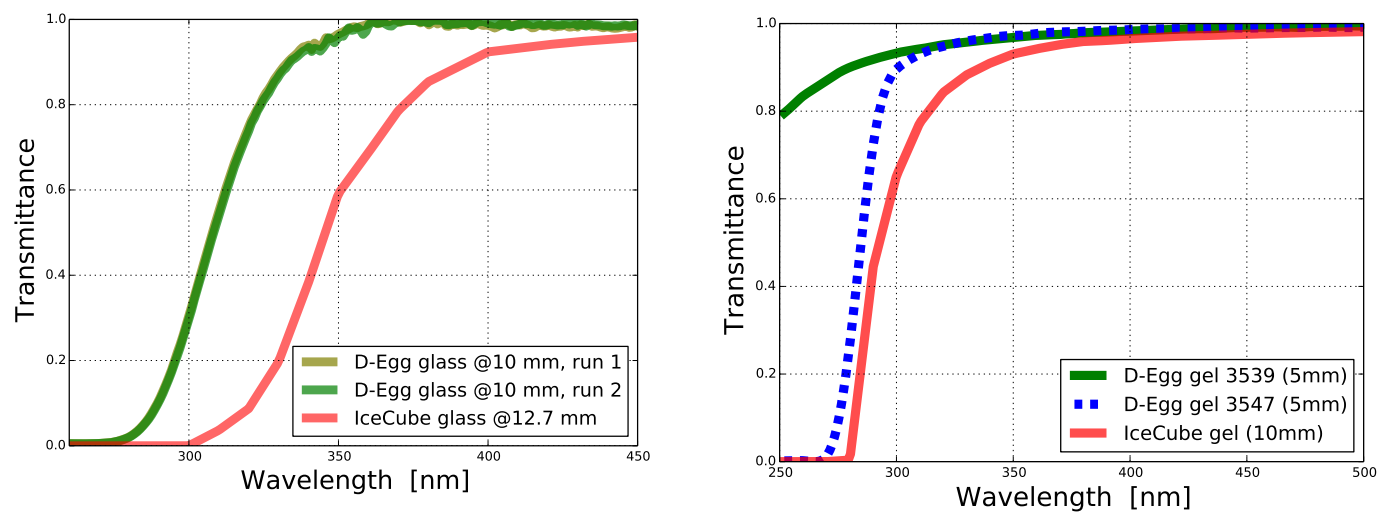

Figure 3: Optical transmittance of the D-Egg housing glass (left) and optical coupling silicone elastomer (right). The glass transmittance was observed as being up to $75 \%$ at $320 \mathrm{~nm}$ and $40 \%$ at $300 \mathrm{~nm}$, a significant improvement over the current IceCube glass.

\begin{tabular}{lcc}
\hline \hline $\begin{array}{l}\text { Sample } \\
\text { thickness })\end{array}$ & $\begin{array}{c}\mathrm{Fe}_{2} \mathrm{O}_{3} \\
\text { by weight [\%] }\end{array}$ & $\begin{array}{c}\text { Transmittance } \\
\text { at } 320 \mathrm{~nm}[\%]\end{array}$ \\
\hline $\mathrm{H}-3-G 1(10 \mathrm{~mm})$ & 0.0095 & 61.7 \\
\hline $\mathrm{H}-3-\mathrm{G} 4(10 \mathrm{~mm})$ & 0.0155 & 39.1 \\
\hline DEgg-1705 $(10 \mathrm{~mm})$ & 0.0061 & 74.4 \\
\hline IceCube $(12.7 \mathrm{~mm})$ & unknown & 10 \\
\hline \hline
\end{tabular}

Table 1: The Fe content and transmittance of glass.

\begin{tabular}{lccc}
\hline \hline Sample & Hardness & $\begin{array}{c}\text { Transmittance } \\
\text { at 300 } \mathrm{nm}[\%]\end{array}$ & Refractive index \\
\hline X-32-3547 $(5 \mathrm{~mm})$ phenylmethyl silicone & Asker C 18 & 91.7 & 1.43 \\
\hline X-32-3539 $(5 \mathrm{~mm})$ methyl silicone & Penetration 35 & 95.5 & 1.4 \\
\hline IceCube gel $(10 \mathrm{~mm})$ & Penetration $\approx 40$ & $\approx 65$ & \\
\hline \hline
\end{tabular}

Table 2: The hardness and transmittance of silicone elastomer.

of glass facing each PMT is reduced for the D-Egg, which has thinner glass. However, further reduction is desirable for two-PMT designs to maintain a low power consumption and data rate. In the glass candidate sample $\mathrm{H}-3-\mathrm{G} 1$, potassium content in the form of $\mathrm{K}_{2} \mathrm{O}$ was artificially substituted to reduce the melting temperature and speed up the glass melting process. The newly developed D-Egg glass, DEgg-1705, replaces $\mathrm{K}_{2} \mathrm{O}$ with $\mathrm{Na}_{2} \mathrm{O}$ to reduce radioactive noise from $\mathrm{K}_{40}$. The $\mathrm{K}_{2} \mathrm{O}$ fraction by weight of the DEgg-1705 was measured as $0.0135 \pm 0.0023 \%$ via XRF, which corresponds to a reduction greater than a factor of two compared to the IceCube glass. XRF also found no scintillation candidate materials, such as a cerium-containing compound. The batch of DEgg-1705 glass was also investigated by using a germanium semiconductor gamma-ray spectrometer (GSGS) for possible contamination by gamma-ray-emitting radioactive material. The GSGS observed a rate of gamma rays from $\mathrm{K}_{40}$ which correspond to $0.74 \pm 0.09 \mathrm{~Bq} / \mathrm{kg}$. While the near-PMT surface thickness is reduced, the glass material is enhanced on the side of the sphere. A more precise estimate of the dark rate of PMTs due to radioactive material in the glass and from the other effect will require improved and detailed GEANT4 simulation and lab measurement. 


\begin{tabular}{lcccc}
\hline \hline Sample & $\begin{array}{c}\mathrm{K}_{2} \mathrm{O} \\
\text { by weight [\%] }\end{array}$ & $\begin{array}{c}\text { Radioactivity } \\
{[\mathrm{Bq} / \mathrm{kg}]}\end{array}$ & $\begin{array}{c}\text { Linear expansion } \\
\text { coefficient }\end{array}$ & Refractive index \\
\hline $\mathrm{H}-3-\mathrm{G} 1$ & 0.73 & 166.4 & $54.23 \times 10^{-7}$ & 1.49 \\
\hline DEgg-1705 & $0.0135 \pm 0.0023$ & $0.74 \pm 0.09$ & $53.98 \times 10^{-7}$ & \\
\hline IceCube (ref. [7]) & $\approx 0.03$ & $\approx 10$ & unknown & \\
\hline \hline
\end{tabular}

Table 3: The potassium content, gamma-ray emitting radioactivity, expansion coefficient, and refractive index of different glass samples.

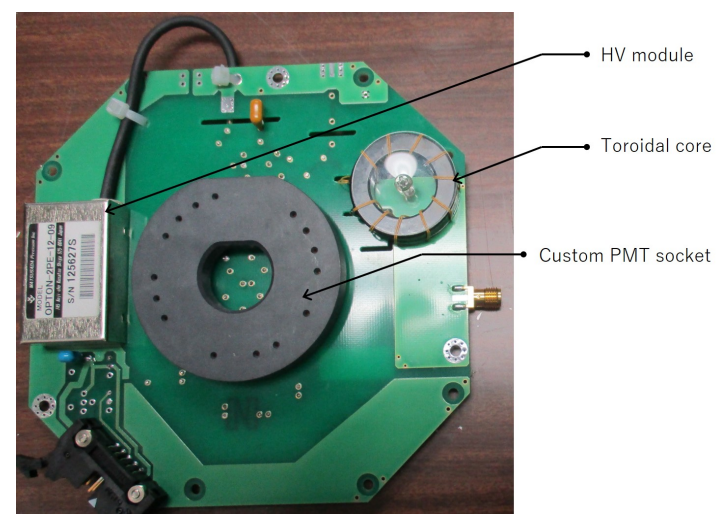

Figure 4: PMT socket with HV board.

\section{HV module}

We developed an HV board for the D-Egg with a divider circuit for the PMT and pulse-shaping and signal-coupling circuits from the cathode-grounded PMT signal using the Matsusada OPTON2PE-12-09 HV module. The stability and characteristics of ripple in the level of $2 \mathrm{mV}_{p p}$ of the HV module were found to be satisfactory. Signal coupling from the PMT DC voltage is achieved using a transformer circuit, as in the IceCube DOM, with a TDK H5C2T31x8x19 toroidal core. A prototype HV base is shown in Fig. 4. The custom PMT space-saving socket enables us to install the HV board to the PMT easily, though in the final design the part may be replaced by direct soldering for cost efficiency.

\section{Magnetic shield}

The performance of the PMT is influenced by a local magnetic field such as that of the Earth. The impact of this effect can be reduced by shielding the PMT with high-permeability materials. There are two options for magnetic shielding for the D-Egg module. One consists of a cage made with mu-metal wires around the photocathode surface PMT, designed similarly to that of the IceCube DOM. The other is made with FineMet ${ }^{\mathrm{TM}}$ foil, which can be wrapped in a conical shape around the PMT neck, similar to that of the Daya Bay experiment optical sensor [8], as shown in the left panels of Fig.5. The performance of each shield was examined by applying a homogeneous magnetic field to a direction perpendicular to the central axis of the PMT. A bare PMT was illuminated by $380 \mathrm{~nm}$ laser picosecond pulser (Hamamatsu) ${ }^{5}$. The detection efficiency $\mu$ (which

\footnotetext{
${ }^{5}$ https://www. hamamatsu. com/jp/en/product/category/1001/5003/M10306-27/index. html
} 

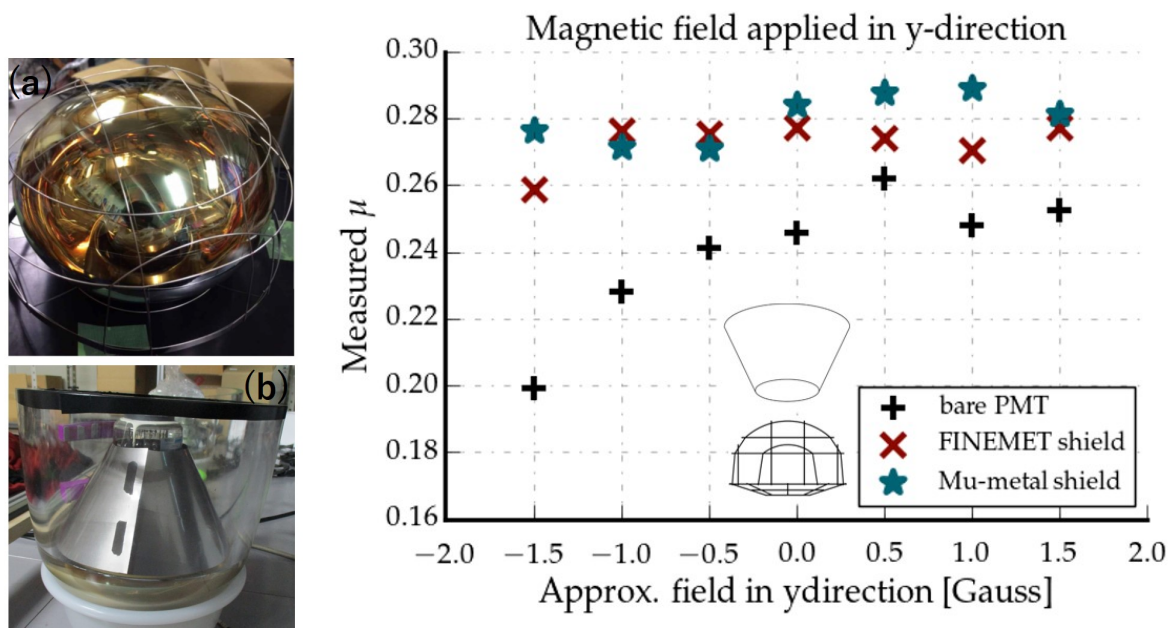

Figure 5: Magnetic shields. Left: (top) mu-metal cage, (bottom) FineMet cone. Right: The light detection efficiency parameter $\mu$ as function of the applied magnetic field.

is proportional to the collection efficiency, quantum efficiency, and light intensity) was measured as a function of the applied magnetic field, as shown in the right panel in Fig. 5. Due to its cost effectiveness and ease of integration, the conical shield is currently considered the default option, although low-temperature performance tests must still be performed.

\section{Data Acquisition and control System}

The readout and communication between surface control units and deep ice optical modules are on a circuit board called the mainboard. The mainboard design is similar to that of the PDOM [4], except for the additional digitizer for the signal from the second PMT. The system architecture of the D-Egg is shown in Fig. 6. The requirements for deep-ice neutrino detection are a power consumption of $\leq 4 \mathrm{~W}$ per module, wide dynamic range from 0.1 of a single photo-electron (PE) signal to more than a thousand PEs, ns-level timing accuracy for PMT pulses, and data transmission rates of greater than $1 \mathrm{Mbps}$. Furthermore, once an optical module is installed in the South Pole glacial ice, it cannot be replaced. Therefore, long-term reliability is one of our highest design priorities. In this contribution, the default design is shown, though the system is still being optimized. The cathode-grounded PMT current signal on the DC high voltage for each PMT is read out through a separate analog front-end that shapes the signal. The shaped signal is digitized by an ADS4149, 14bit, 250-MSPS ADC with $265 \mathrm{~mW}$ of total power for each PMT. Digital processing is performed in a field-programmable gate array (FPGA). A single FPGA (Cyclone V 5CEFA7F23C7N) takes the continuously digitized waveforms from two digitizers and applies flexible triggering, such as a voltage threshold or local coincidence between the signal from two PMTs. The mainboard also contains the HV control system with 8-bit digital-to-analog converter (DAC) and communication hardware to interface to the communication card on the surface counting house, including timing calibration and power management.

\section{Summary}

All necessary components of the D-Egg IceCube-Gen2 optical module have been designed, 


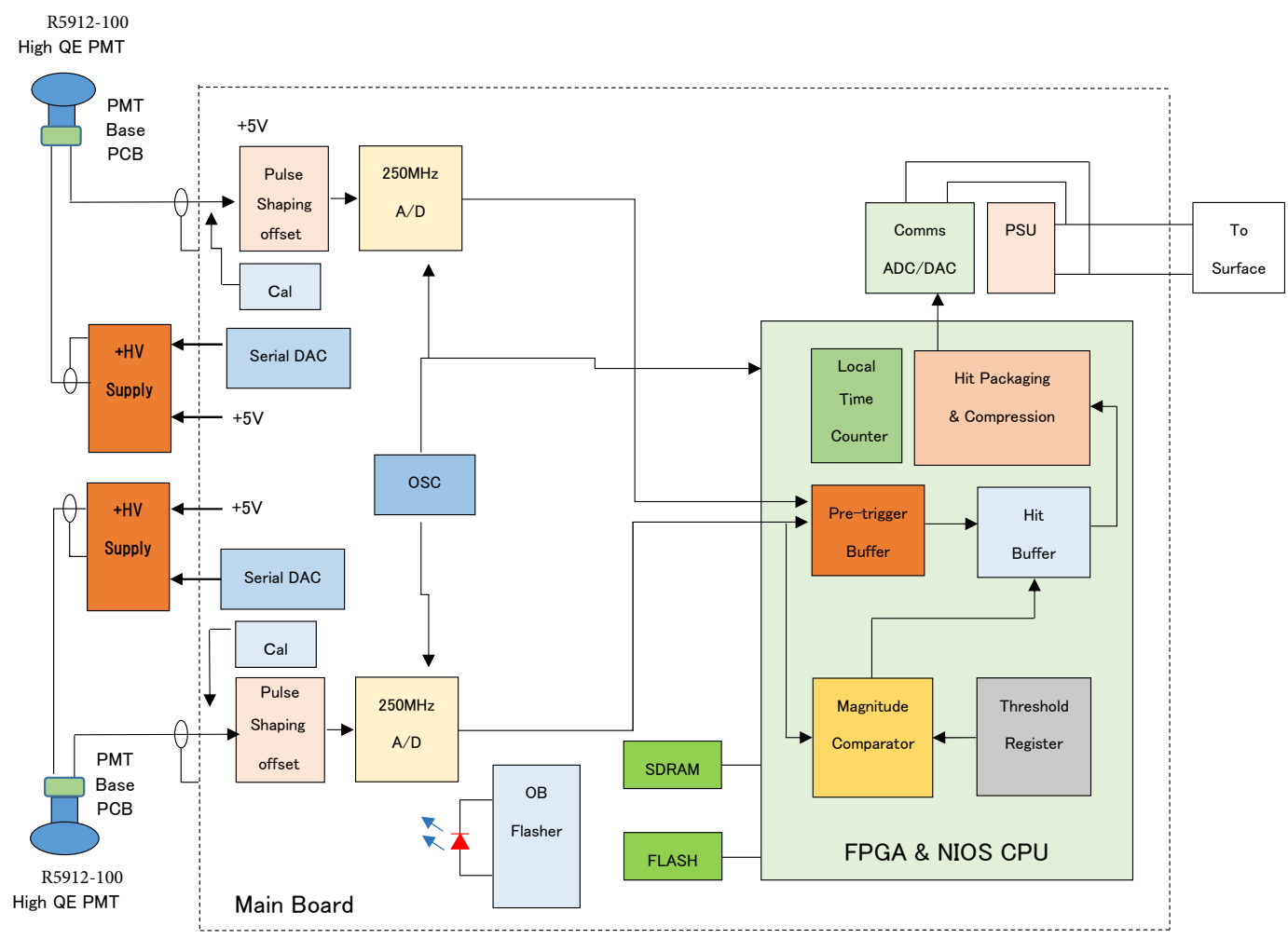

Figure 6: Mainboard block diagram. This low power consumption mainboard is responsible for readout and digitization of PMT signals, communication with surface control units, monitoring and calibration. Two digitizers, one for each PMT, continuously digitize waveform signals from PMTs, to be processed in an on-board FPGA.

and the second batch of prototype modules is being produced. D-Egg's elongated shape and upward-facing PMTs are new to IceCube, and its cost-effective construction of a deep-ice array of optical sensors establishes good angular resolution for all directions currently being studied [3]. While maintaining the successful design architecture of the IceCube DOM, D-Egg has successfully improved upon several of its major components.

\section{References}

[1] IceCube-Gen2 Collaboration, PoS (ICRC2017) 934 (these proceedings).

[2] IceCube-Gen2 Collaboration, POS ( ICRC2017) 1040 (these proceedings).

[3] IceCube-Gen2 Collaboration, PoS ( ICRC2017) 1038 (these proceedings).

[4] IceCube-Gen2 Collaboration, PoS ( ICRC2015) 1148 (2015); P. Sandstrom and IceCube-PINGU Collaboration, AIP Conference Proceedings 1630, 180 (2014) (http://doi.org/10.1063/1.4902801).

[5] IceCube-Gen2 Collaboration, PoS ( ICRC2017) 1047 (these proceedings).

[6] IceCube-Gen2 Collaboration, PoS ( ICRC2017) 1052 (these proceedings).

[7] IceCube Collaboration, M. G. Aartsen et al., JINST 12 (2017) P03012.

[8] P. DeVore et al., Nucl. Instr. and Meth. A 737 (2014) 222. 